\title{
ANÁLISIS INTRODUCTORIO DE LAS FORMAS DE CONCENTRACIÓN DE LA INFORMACIÓN EN EL SOFTWARE SOCIAL Y LAS RELACIONES DE PODER
}

\author{
ANÁLISIS INTRODUCTORIO DE LAS FORMAS DE \\ CONCENTRACIÓN DE LA INFORMACIÓN EN EL \\ SOFTWARE SOCIAL Y LAS RELACIONES DE PODER
}

\author{
ALFREDO CALDERÓN S. ${ }^{1}$ \\ OSWALDO ESPINOSA ${ }^{2}$ \\ TATIANA NOBOA M. ${ }^{3}$
}

\footnotetext{
${ }^{1}$ Pontificia Universidad Católica del Ecuador, Facultad de Ingeniería, Quito, Ecuador (acalderon@puce.edu.ec).

2 Pontificia Universidad Católica del Ecuador, Facultad de Ingeniería, Quito, Ecuador (loespinosa@puce.edu.ec).

${ }^{3}$ Universidad de las Fuerzas Armadas - ESPE, Departamento de Ciencias de la Computación, Quito, Ecuador (tknoboa@espe.edu.ec).
} 
illl|l 208

208 


\section{ANÁLISIS INTRODUCTORIO DE LAS \\ FORMAS DE CONCENTRACIÓN DE LA INFORMACIÓN EN EL SOFTWARE SOCIAL Y LAS RELACIONES DE PODER}

\section{ANÁLISIS INTRODUCTORIO DE LAS FORMAS DE CONCENTRACIÓN DE LA INFORMACIÓN EN EL SOFTWARE SOCIAL Y LAS RELACIONES DE PODER}

Alfredo Calderón S., Oswaldo Espinosa V., Tatiana Noboa M.

Palabras clave: computación y sociedad, tecnología, información, redes sociales, buscadores, big data (grandes datos), Google, Twitter, Facebook, informática social

Keywords: computing and society, technology, information, social networks, search engines, big data, Google, Twitter, Facebook, social computing.

\section{RESUMEN}

El presente artículo visibiliza que como nunca antes, en la humanidad y a pesar de los grandes adelantos tecnológicos, la información se encuentra centralizada en grandes corporaciones, que utilizan dicha data en función de sus propios intereses. Lo anteriormente citado, a pesar parecer sobreentendido a nivel no formal, requiere de un ordenamiento teórico que además presenta elementos del estado del arte de las formas de concentración de la información en el software social. Por tal motivo al inicio se mostrará que es Big Data desde una perspectiva técnica y de fácil entendimiento, seguidamente se analizarán a 
tres corporaciones que manejan estos grandes volúmenes de datos desde la visión de sus creadores, estas son Google, Twitter y Facebook, se profundizará mediante un análisis de casos en donde se podrá apreciar sus beneficios pero a la vez sus objetivos y por último se presentarán conclusiones de este trabajo tanto generales como filosóficas.

\section{ABSTRACT}

The present article makes visible as never before, in the humanity and in spite of the great technological advances, the information is centralized in large corporations, which use this data according to their own interests. The aforementioned, seems to be understood on a non-formal level, however requires a theoretical order that also presents elements of the state of the art of the forms of concentration of information in social software. For this reason, at the beginning will show that it is Big Data from a technical perspective and easy to understand, then it will analyze three corporations that handle these large volumes of data from the vision of its creators, these are Google, Twitter and Facebook, will be deepened through an analysis of cases where you can appreciate its benefits but also its objectives, and finally, will be presented conclusions of this work, both general and philosophical.

\section{INTRODUCCIÓN}

Hoy en día se utilizan a diario herramientas tecnológicas de la comunicación y de la información (Tics), como Google y Facebook que se han convertido en muy familiares a los usuarios de Internet, sin embargo, juntos manejan un volumen de información como nunca antes ha tenido la humanidad, por lo que se han desarrollado nuevas tecnologías que facilitan su manejo, pero este avance tecnológico suscita nuevos escenarios como el de la centralización de la información que puede derivar en el uso y abuso de esta información por parte de estas empresas privadas, o quienes por un medio u otro puedan acceder a esa información.

Por esta razón en el presente artículo se realiza un análisis introductorio de las formas de concentración de la 
información en el software social, con la intención de analizar estos puntos, y probar que estas empresas pueden tener el poder de usar la información de sus usuarios para obtener poder y de esta manera lucrar sin importar comportamientos éticos.

Para ello se buscará identificar cual es la visión de sus creadores y con esto poder entender el funcionamiento en sí de estas corporaciones.

Adicionalmente, el presente estudio pretende contribuir con equilibrar o llevar al centro el entendimiento de la labor de científicos de las ciencias de la computación como aportadores reales de ideas para el bien común, mediante técnicas desarrolladas en el área y no solo como constructores de herramientas para uso de terceros, proveyendo la visión del estado del arte de la cuestión desde un punto de vista filosófico y no solamente técnico, creando una serie de cuestionamientos que todo cientíico del área debería plantearse.

\section{BIG DATA}

La cantidad de datos que arrojan los diferentes dispositivos informáticos, páginas web, correos, buscadores, redes sociales, geolocalizadores, la mencionada "Internet de las cosas", aplicaciones públicas y privadas, entre muchos otros, han hecho que su procesamiento de datos sea imposible por los métodos tradicionales de manejo de la información, tales como "bases de datos relacionales". Por este motivo han aparecido nuevas tecnologías que permiten tanto el manejo de estos volúmenes de datos así como, el análisis de las relaciones que puede existir entre estos grandes volúmenes de datos y su posterior "toma de decisiones" para así convertir toda esta "enorme data" en in- formación relevante. En el presente artículo no se analizan las tecnologías que sirven para procesar los grandes volúmenes de datos, sino más bien los efectos de dichos usos, específicamente por las grandes empresas proveedoras de contendidos.

Para visualizar la información que se produce, se puede observar lo siguiente de acuerdo con la Web oficial de la Unión Europea (Unión Europea, 2015) y direccionada por el periódico El País de España ( Van den Berg):

- 1700 nuevos billones de bytes por minuto, equivale a $360000 \mathrm{dvd} \mathrm{o}$ sea 6 Megabytes por día de una persona. 
- Más de un billón de consultas en google por día.

- 250 millones de tuits por día.

- 800 millones de actualizaciones en Facebook por día.

- 60 horas de videos subidos por minuto en YouTube

Entre los beneficios que se pueden obtener usando esta tecnología podrían ser listados los siguientes: (Van den Berg):

- Predecir tormentas, huracanes, terremotos, erupciones volcánicas o tsunamis.

- Monitorear la evolución del cambio climático.

- Conocer el estado de los océanos.

- Discernir el comportamiento de los animales o realizar el seguimiento de especies amenazadas.

- Relacionar contenidos entre muchos libros y encontrar posibles relaciones nunca antes ubicadas.

- Para vender más, de la forma más óptima y personalizada posible.

\section{Google y Facebook, los más visitados}

De acuerdo con los datos suministrados por Alexa Internet Inc'1, los sitios más visitados a marzo del 2015 son Google y Facebook en su orden, ver tabla 1:

Tabla 1

\begin{tabular}{|c|c|}
\hline Ranking & Sitio \\
\hline 1 & Google.com \\
\hline 2 & Facebook.com \\
\hline 3 & Youtube.com \\
\hline 4 & Baidu.com \\
\hline 5 & Yahoo.com \\
\hline 6 & Wikipedia.com \\
\hline 10 & Twitter \\
\hline
\end{tabular}

Existe otra empresa llamada "Similar web"2que también ubica a los sitios por ranking en donde actualmente Facebook es el número uno y Google es el segundo, pero si se toma en cuenta que YouTube se encuentra tercero en las dos mediciones y dicho sitio es de propiedad de Google, se tiene nuevamente que Google se encuentra en primer lugar.

\footnotetext{
${ }^{1}$ Es una subsidiaria de la compañía Amazon.com con base en California. Es conocida por operar el sitio web alexa.com que provee información acerca de la cantidad de visitas que recibe un sitio web y los clasifica en un ranking

${ }^{2}$ Es una compañía de tecnología de la información cofundada en 2007 y actualmente de propiedad de Google. Su sede se encuentra en Londres, Reino Unido y realiza trabajos en Inteligencia de Negocios.
} 


\section{TWITER}

Es una red social de "mensajes cortos" creada en San Francisco, por Jack Dorsey (nacido en 1976) en marzo de 2006. También es Ilamado el "SMS" del Internet, en alusión a los mensajes de celular ya casi no utilizados.

No está claro de quién fue la idea que gestó esta red social, se indica que esta vino de una compañía llamada Odeo, pero que no tuvo éxito debido a un producto similar por parte iTunes, por lo que uno de sus creadores vendió su parte en 7000 dólares por desavenencias entre ellos.

La siguiente expresión identifica el para qué de Twitter:

"En Facebook encontraremos a quienes estudiaron con nosotros, y en Twitter encontramos a aquellos que nos hubiera gustado que estudiaran con nosotros" (Gámez, 2012)

Con esta frase se quiere decir, que en twitter se encuentra a las personas que de una u otra forma se asemejan más en su forma de pensar o sentir, ya que "seguimos" o nos "siguen" personas que se alinean a una misma manera de ser o de percibir el mundo.

La publicidad es la principal fuente de ingresos de Twitter. Los formatos que comercializa son los tuits patrocinados, las tendencias patrocinadas y las cuentas patrocinadas. Durante el primer semestre de 2013, Twitter ingresó 221 millones de dólares en concepto de publicidad (su facturación global en 2012 fue de 316 millones de dólares). (Orihuela, 2013)

Además de la publicidad, Twitter vende el acceso a sus datos, lo que representa un $13 \%$ de sus ingresos (32 millones de dólares durante el primer semestre de 2013). (Orihuela, 2013)

Todos los mensajes son públicos y se pueden trabajar con varios universos a la vez. Esto quiere decir que absolutamente todo lo que se dice, se "postea" o se "retwitea" sea esto palabra u objeto multimedia será siempre de uso público (a diferencia de Facebook que lo que se ubica es únicamente para los amigos, familiares o persona). Al decir "universos" significa que se puede ubicar cualquier tweet por diversas formas estos son los "hashtags" los que pueden ubicar cualquier tweet.

La credibilidad de los gurús por parte de los usuarios es elevada, sobre todo cuando hablan de un tema que dominan, aunque siempre hay grupos que no lo aceptan debido a la idiosincrasia de Twitter. En una red entre iguales no tiene sentido que exista alguien por "encima" de uno mismo.

El poderío de esta red social reside básicamente en que cualquier perso- 
na de forma ubicua (tiempo y lugar) puede indicar, decir, opinar, etc., a cualquier persona, no importa lo grande, distante o famosa que parezca. Logra cambiar la forma de pensar, como diría John Perry Barlow ${ }^{3}$ se está pasando de un mundo "Monoteísta", es decir en la creencia de un solo Dios, a uno "Panteísta" (todo es Dios), por cuanto se está dejando de pensar y crear organizaciones de manera "jerárquica", ya que en este modelo, siempre existirá alguien que esté en lo alto, y para llegar a él, necesitaremos de un intermediario (por ejemplo un santo o un sacerdote en la Iglesia Católica o un subgerente en el ámbito empresarial), mientras que en el modelo "red" llamémosle Panteísta, todos somos iguales, no existen jerarquías, por cuanto en la red cada una de las personas se pueden comunicar con cualquier otra persona sin importar qué cargo ocupe dentro de cualquier organización, sin intermediarios. Según él, la culpa la tiene Abraham, ya que a través de él se crearon varias religiones monoteístas.

\section{GOOGLE}

Esta empresa desde la creación de su algoritmo único de búsqueda basada en las preferencias de los propios usuarios marcó un cambio radical en la forma en como encontramos la información que buscamos, ha creado un sinnúmero de servicios para sus usuarios y a todos estos servicios los ha puesto de manera "gratuita" para su uso, tales como: Gmail (servicio de mensajería), hoy nos obligan a usarlo cuando utilizamos su sistema operativo (Android) para celulares, tabletas y más, Drive (almacenamiento en la nube con mucho espacio en disco gratuitamente), Maps
(Georeferenciación), Traductor, Contactos, entre otros. Existe una máxima que dice que cuando es gratis significa que uno es el producto, en sí esta máxima no se aleja de la realidad. Google posee uno de los mejores modelos para mercadear productos, este se basa en la búsqueda de las preferencias de los usuarios, para de esta manera presentar a su usuario los mejores productos o servicios que serán de la preferencia del mismo, sea esto por medio de su propia página web o presentando en los correos publicidad correctamente dirigida a cada usuario.

\footnotetext{
${ }^{3}$ Nacido en Jackson Hole, Wyoming (Estados Unidos), nacido el 3 de octubre de 1947, es un poeta, ensayista y ganadero retirado estadounidense. Es cofundador de la Electronic Frontier Foundation junto con otros activistas de los derechos digitales.
} 
Al igual que las grandes corporaciones como Microsoft que en su tiempo marcaron el avance de la tecnología (su creador Bill Gates tenía como visión tener 9 de cada 10 computadores con software de Microsoft y claro que lo consiguió), la meta de su principal creador, Larry Page, es "organizar y hacer accesible la información en el mundo", así como ejemplo de esta visión son sus productos Google Books o Google News.

Con Google Books, Google ha desarrollado el proyecto de "escanear" los libros de las bibliotecas de las mejores universidades del mundo, por ello realizó convenios con muchas universidades para digitalizar dichas bibliotecas, para ello desarrolló su propia tecnología de escaneo de libros, la que no ha hecho conocer, suena contradictorio que al ser la que democratizará el conocimiento del mundo, no democratice el conocimiento que utilizó para desarrollar su propia metodología. El proyecto lo han Ilamado Alejandría 2.0, hace alusión a la gran biblioteca de la antigüedad. Google creó un problema ético y otro legal. El ético es que al ser una empresa privada, ¿podrá esta empresa tener todo este conocimiento?, ¿qué pasaría en el supuesto que como toda obra humana termine o quiebre, quién pasaría a poseer esta gran data?. Con respecto al problema legal es que nunca negoció con los autores ni con las editoriales de las obras, bastó con negociar con las poseedoras de los libros: las bibliotecas, esto generó en muchos juicios, pero sin embargo, por el poder que tiene esta trasnacional continúa con su proyecto. ¿Qué tanto es legal que intente ser memoria del mundo y por lo tanto poseer el monopolio del acceso a la información?.

Uno de los capítulos que mejor muestra el poder de Google viene de Bélgica (2006), donde un grupo de periódicos demandó a Google por utilizar Google News, sitio de Google que almacena los titulares de muchos periódicos a escala mundial. La demanda consistía en justamente eso, tomar los titulares sin ningún permiso y ofrecer dicho contenido en otro sitio distinto al de los periódicos, por lo que los periódicos aducían que poseen anunciantes que quieren que sus avisos sean vistos al consumir las noticias que ofrecen en sus portales, es decir que existía un daño económico además de la violación de la propiedad intelectual. Google aducía que dicha información la toma pero no gana absolutamente nada con hacerlo, es decir que no existe un objetivo económico y por lo tanto es legal. El juez que tomó la causa falló a favor de los medios de comunicación. Seguidamente Google acató el fallo, pero tomó represalias eliminando esos periódicos de su motor de búsqueda. Los dueños de estas empresas vieron 
como se desplomaban sus visitantes de un día al otro. Los diarios tuvieron que aceptar la derrota y se fueron por la menor pérdida, es decir aceptar la presencia de Google News. Este es una de las historias que ejemplifica la relación entre tecnología y poder. (Suárez Sánchez, 2012).

Google ha continuado con su "visión" de ser el poseedor del "conocimiento del mundo" con más productos y/o servicios gratuitos como:
Google Sites: Aplicación en línea que permite crear un sitio web de manera segura y ágil.

Google Calendar: Agenda y calendario electrónico, casi todos los usuarios de "Android" lo usan. Ha tenido complicaciones en la privacidad que se cruzan las agendas de varias personas

Google Traductor: Permite traducir a más de 50 idiomas de forma casi instantánea.

\section{FАСЕВООК}

El creador de Facebook, Mark Zuckerberg, nacido el 14 de mayo 1984, cuando lo creó tenía la edad de 22 años, al igual que Larry Page (google) es una persona ambiciosa que tiene clara la visión de lo que quiere de su empresa, en palabras del propio Zuckerberg: "Crear un servicio que recoja en su totalidad el contacto humano", en otras palabras, trazar la "gráfica social" al conjunto de las relaciones que posee la gente. Este es un concepto académico del análisis de redes sociales, todos están conectados por una red de relaciones, unos son directos y fuertes, otros tenues y débiles, estos son los grados de separación que tienen todos con todos, el mismo que llega hasta un máximo de seis saltos. Se puede imaginar un diagrama de las conexiones interpersonales, ese es el mapa social. Zuckerberg habla sobre trazar, englobar y reproducir todo el mapa social del mundo. (Discovery Chanel, 2012)

Se puede decir que Facebook es el "Internet de las personas", posee lo principal del accionar humano: "sus relaciones", ya que el ser humano es en sí un ser social. Esta es la información que no posee Google quienes han desarrollado intentos pero no han tenido el éxito que ha experimentado Facebook (Google plus). Este es otro ejemplo de gran almacenamiento de datos y que es imposible guardarlo y manipularlo de las maneras tradicionales. Ahora Facebook sabe lo que le gusta al usuario, y con esta información se puede direccionar cualquier 
producto o servicio que se quieran promocionar, pero quien realiza la promoción, publicidad o invitación a un evento, es el hermano, primo, amigo, o el amigo del amigo quien llega a "mercadear". Es decir Facebook inventó un nuevo modelo de publicidad. No es lo mismo que una marca publicite un producto o servicio (al estilo de la televisión), ahora son los círculos sociales, quienes tienen parecidas pasiones a las que uno tiene, y son ellos quienes te sugieren el uso de este producto o servicio. Esto es lo nuevo que ha producido Zuckerberg.

¿Ahora qué puede hacer una empresa con esta información, a más de mercadear un producto? El siguiente experimento realizado por Facebook cuenta nuevamente la relación entre tecnología y poder que poseen estas empresas (Sánchez, 2014):

El experimento, realizado en colaboración con dos universidades de Estados Unidos (Cornell y San Francisco), duró una semana (del 11 al 18 de enero de 2012), el cual consistía en enviar noticias tanto positivas como negativas a grupos de usuarios segmentados para el efecto (689.003 perfiles).

A los usuarios que observaron historias positivas se encontró que estos son menos propensos a escribir un mensaje negativo. Y viceversa. Luego, el equipo de expertos liderado por los doctores Adam Kramera, de Facebook; Jamie Guillory, de la Universidad de California y Jeffrey Hancock, de la Universidad de Cornell, analizaron cerca de tres millones de comentarios y emplearon su propio código para caracterizar su lenguaje como positivo o negativo (forma típica de manejo de Big Data para poder procesar grandes volúmenes de datos).

Al final se concluyó:

1. La influencia social dictamina el estado de ánimo, y

2. Los estados emocionales se pueden transmitir entre personas que no están juntas físicamente y sin que interactúen entre ellas.

A más de lo interesante del estudio, fue que las personas objeto de este experimento, nunca fueron consultadas si querían ser parte del mismo. Se desató una polémica por este "inconveniente" $y$ Facebook pidió disculpas públicas.

El problema en sí, no fue el "permiso", el problema mayor fue que se comprobó que se puede manipular el estado emocional de las personas a través de las redes sociales. 


\section{CONCLUSIONES}

En estos tiempos han surgido nuevas empresas producto del desarrollo tecnológico que se va experimentando y que a su vez han generado dependencia a las mismas, esto ha hecho que estas nuevas empresas hayan acumulado poder y a la vez grandes fortunas. Como es el caso de estas dos empresas analizadas, Google y Facebook. Es decir se ha encontrado una relación entre tecnología - dependencia - poder y acumulación de capital. Pero esta no necesariamente es la regla, ya que también han surgido empresas que han generado dependencia a través de la tecnología, y también poseen un cierto poder sin acumulación de capitales, este es el ejemplo de la corporación Wikipedia, con su creador y persona altruista, como le han catalogado, Jimmy Wales. (Martínez, 2012)

Pero los creadores de estas empresas son gente joven, inteligentes, ambiciosos y con una visión clara de lo que quieren, además de estar en el sitio donde se hace todo posible: "El valle del silicio". Alguna vez viajaron juntos Robert Murdoch 4 y Zuckerberg, luego de la conversación le preguntaron a Murdoch que le pareció y contestó que es un "tipo interesante, muy joven pero con mucha suerte, su modelo de negocios caerá". Un año más tarde Facebook cotizaba en la bolsa y uno de los mejores periódicos de Murdoch quebraba y a la vez compraba a la empresa MySpace (competidora directa de Facebook) por 580 millones de dólares (2005), más tarde la vendería (2011) en 35 millones de dólares. Esto muestra que quien mejor conoce a la gente, específicamente a la gente joven son los nuevos jóvenes.

¿Pero que hace que estos sitios sean del gusto popular? No hay que olvidar que actualmente se vive en el mundo de Internet y que es la "sabiduría" o la "estupidez" de masas quienes escogen que sitio es importante, podría darse, de hecho es una realidad que exista más gente viendo videos "tontos" en YouTube que leyendo en el sitio Web del New York Times. Quien podría dar respuesta a esto es uno de los creadores de Napster ${ }^{5}$ quien al hacerlo dijo que fue muy excitante y a la vez placentero encontrarse con gente que tenía las mismas pasiones que uno. (Discovery Chanel, 2012)

Big Data, como toda creación humana es una herramienta más, pero una

\footnotetext{
${ }^{4}$ Nacido en Melbourne, Australia, 11 de marzo de 1931, es el magnate actual de las comunicaciones. Dueño entre otros de Fox.

${ }^{5}$ Fue un servicio de distribución de archivos de música creado por Sean Parker y Shaw Fanning (en formato MP3) basado en el intercambio de música entre iguales.
} 
de las más poderosas que ha inventado el ser humano que en manos de gente o corporaciones que tengan un interés económico como prioridad es un peligro, porque antepondrán su interés a un principio, llegado cualquier momento extremo.

Surge la pregunta si Facebook, Twitter y Google son empresas tecnológicas o son empresas dedicadas al marketing, ya que sus ingresos vienen de la segunda forma.

Esto lleva a reflexionar si; ¿es ético dejar que el poder de restringir el acceso al conocimiento humano así como sus relaciones estén en empresas privadas?, es más ¿es bueno para la humanidad almacenar y gestionar la información? ¿se podría detenerlas?, ¿se puede salir de ellas?

Se debe cuestionar si en realidad se tiene la suficiente información como para creer que se conoce todo acerca de determinado tema, o se debe entender se tiene acceso solamente a aquello que se visualiza, o aquella información que alguien tomó la decisión de publicar espontáneamente o por intereses varios, estas herramientas lo que han hecho es crear la ilusión de completitud de la información, dado que lo publicado no es todo lo que existe del tema sino más bien restringido de varias formas. No todo lo que existe está, ni todo lo que está es verdad.
Tanto los avances cientíicos como tecnológicos siempre han estado un paso adelante de la deliberación filosófica, la pregunta es, como en algún momento lo dijo Mario Bunge: " ¿En que puede interesar estos avances a los filósofos?", se pensaría que de este extracto surgen muchas preguntas que deben ser reflexionadas y respondidas por los mismos, como las formuladas en el anterior punto.

El ejemplo de utilización de Big Data propuesto en este trabajo muestra que la teoría no es ajena a la tecnología, ya que las ciencias que motivaron su aparecimiento son las que tienen que ver con búsqueda de patrones para la toma de decisiones, estudiadas particularmente en las ciencias computacionales y en las administrativas en los grandes volúmenes de datos.

La tecnología ha invadido el diario vivir del ser humano, de forma visible al inicio, como es el caso de las computadoras y se ha ido incrustado cada vez de manera menos visible, se habla mucho de nanotecnología, de "Internet de las cosas", de "Internet de los órganos", pero no solo es eso, también es capaz, como es el caso de Big Data, de incidir en los comportamientos sin que apenas se note a pesar de estar desconectados.

En la actualidad existe una esfera pública "offline" y "online". Hasta la aparición de las redes sociales, la esfera 
pública offline fue la dominante, en donde los medios de comunicación ejercían su poder sin ninguna competencia. Los ciudadanos tenían un papel pasivo hacia los hechos. Hoy en día gracias a estas herramientas como Twitter y Facebook el accionar de los ciudadanos es una posición incrédula y crítica con respecto a los mensajes o líderes sean quien fuera o de donde viniera.

Han surgido nuevas formas de "democratizar el conocimiento" o en otras palabras de la "apropiación social del conocimiento" para el beneficio de la sociedad en su conjunto pero al mismo tiempo para el lucro de unos pocos, como es el caso de las empresas analizadas en el presente trabajo, pero todo este conocimiento que se podría calificar como bueno, malo o también basura, depende de lo que se quiera encontrar, presenta nuevas inquietudes éticas y morales, como es el manejo que se quiera dar a esta información y/o conocimiento.

Estas tecnologías de uso común han venido con una nueva generación humana que ha cambiado los valores éticos y morales, basta con hacer una pregunta a ellos: ¿Han comprado música los últimos años?, sino lo han hecho, como es seguro su respuesta, en su mayoría, ¿sintieron que perjudicaban al autor al "bajarse" o "compartirla"?, es seguro que nunca les pasó por la mente esta situa- ción, es decir que tenemos una nueva generación que presenta nuevos problemas éticos como legales y que a la vez se tenga que repensar tanto los códigos como las leyes que existen actualmente, ya que no es posible que se criminalice a toda esta generación.

En estos ámbitos, ¿cómo se puede regular el ciberespacio?, dejar que las empresas y/o gobiernos monopolicen este espacio, ¿crear leyes que lo controlen? Dada la complejidad del tema se considera que solo se pueden regular por ética y tecnología, dejando que los verdaderos actores: usuarios, proveedores de contenidos, creadores de información, proveedores de Internet, activistas de los derechos digitales creen sus propios códigos de conducta. En el ciberespacio ya no existen naciones (el problema de la Gobernanza de Internet actualmente está en desarrollo).

Las nuevas formas de poder han trasmutado, ya no son autoritarias, ahora se ocultan, pero igualmente el usuario se postra ante ella pero de forma tanto consciente como inconsciente, tanto por la utilidad como por la novelería de la tecnología y de esta forma se entrega toda la información, en este caso a las empresas que proveen contenidos.

Pero que ha producido el desarrollo de estas tecnologías: ¿el afán del ser humano por comunicarse?, ¿la democratización del conocimiento?, ¿el 
lucro?, ¿la innovación? o ¿todas ellas a la formación y/o conocimiento que seguirá vez? Pero lo que si es cierto es que estas siendo aprovechado por estas empresas han mejorado y a la vez creado necesi- tecnológicas que han centralizado a nidades, por ello se sigue produciendo in- vel mundial la información. 


\section{BIBLIOGRAFÍA}

Van den Berg, E. (s.f.). El País. Recuperado el 2015 de marzo de 2015, de El País: http://elpais.com/ elpais/2015/03/26/buenavida/1427382655_646798.html

Discovery Chanel. (2012 de junio de 2012). El futuro digital. Recuperado el 10 de noviembre de 2015, de El futuro digital: https://www. youtube.com/watch?v=41030cktwC8

Gámez, D. (2012). Twitter Cómo 140 caracteres pueden ayudarle a mejorar su presencia on line. Barcelona, España: PROFIT Editorial.

Martínez, C. (2012). Wikipedia Inteligencia Colectiva en la red. Barcelona, España: Profit Editorial.

Orihuela, J. L. (5 de noviembre de 2013). Ecuaderno. Recuperado el 11 de octubre de 2015, de Ecuaderno: http://www.ecuaderno. com/2013/11/05/el-modelo-de-negocio-de-twitter/

Sánchez, J. (01 de julio de 2014). ABC
Tecnología. Recuperado el 14 de noviembre de 2015, de ABC Tecnología: http://www.abc.es/ tecnologia/redes/20140630/ abci-facebook-manipula-algoritmo-noticias-positivas-negativas-estudio-psicologico-201406300950.html

Suárez Sánchez, A. (2012). Desnud a Google, La inquietante realidad que no quieren que conozcas. Barcelona, España: Grupo Planeta.

Unión Europea. (31 de marzo de 2015). Web oficial de la Unión Europea. Recuperado el 12 de noviembre de 12, de Web oficial de la Unión Europea: http://europa.eu/index_es.htm

Wikipedia. (s.f.). Wikipedia: Sitios Web mas visitados. Recuperado el 1 de noviembre de 2015, de Wikipedia: https://es.wikipedia.org/wiki/ Anexo:Sitios_web_m\%C3\%A1s_ visitados 